\title{
Análisis Del Comportamiento De Las Precipitaciones En Quevedo - Ecuador, Para La Planificación De Cultivos
}

\author{
Oscar Caicedo-Camposano, Profesor Investigador \\ Dalton Cadena-Piedrahita, Profesor Investigador \\ Luis Alcívar-Torres, Profesor Investigador \\ Adela Veloz-Paredes, Experta en Informática \\ Franklin Montecé-Mosquera, Profesor \\ Universidad Técnica de Babahoyo, Ecuador
}

doi: 10.19044/esj.2016.v12n33p212 URL:http://dx.doi.org/10.19044/esj.2016.v12n33p212

\begin{abstract}
The work was performed with the aim of knowing the rainfall of the city of Quevedo in Ecuador, to improve the calendars of short-cycle crops, since this information is commonly required by farmers in the area and agricultural professionals who work as technical advisers.

Processing a series of recordings of rainfall of 35 years belonging to the Pichilingue Weather Station of the National Institute of Meteorology and Hydrology located on the premises of the National Agricultural Research Institute, the methodology applied was focuses on the determination of statisticians as the average, Standard Deviation, Variance, coefficient of variation, skewness and kurtosis coefficient; then based on the average are determined wet and dry periods the frequency and the intensity with which they occur. That also was detected in this town from 1980 to 2014 there were 10 dry periods and nine wet periods, which are presented with a frequency of 3,8 and 3,6 years respectively. Based on the results, the wet season is defined between the months of December to May and the dry season from June to November. The foil available average rain accumulated determined for the wet and dry periods has the following values: $953,3 \mathrm{~mm}, 2924,2 \mathrm{~mm}$ and an average of 2216,3 $\mathrm{mm}$. Short-cycle crops of the area should be planted since the month of December to be exploited all the rain of the wet season.
\end{abstract}

Keywords: Characterization of precipitation, dry periods, wet periods 


\section{Resumen}

El trabajo se efectuó con el objetivo de conocer el régimen de precipitaciones de la ciudad de Quevedo en Ecuador y de esta manera contribuir al mejoramiento de los calendarios de cultivos de ciclo corto, puesto que, esta información es comúnmente requerida por agricultores de la zona y profesionales agrícolas que laboran como asesores técnicos. Se procesó una serie de registros de precipitaciones de 35 años pertenecientes a la Estación Meteorológica Pichilingue del Instituto Nacional de Meteorología e Hidrología situada en los predios del Instituto Nacional Autónomo de Investigaciones Agropecuarias, la metodología aplicada se centra en la determinación de estadísticos como la media, desviación estándar, varianza, coeficiente de variación, coeficiente de asimetría y coeficiente de curtosis; luego con base en el promedio se determinan periodos secos y húmedos, la periodicidad y la intensidad con que estos ocurren. Se detectó que en esta localidad desde 1980 a 2014 ocurrieron 10 periodos secos y nueve periodos húmedos, los cuales se presentan con una periodicidad de 3,8 y 3,6 años respectivamente. Fundamentado en los resultados, la estación húmeda queda definida entre los meses de diciembre a mayo y la estación seca de junio a noviembre. La lámina disponible promedio de lluvia acumulada determinada para los períodos seco y húmedo tiene los valores siguientes: 953,3 mm, 2924,2 mm y un promedio de 2216,3 mm. Los cultivos de ciclo corto de la zona deberían sembrarse desde el mes de diciembre para que sea aprovechada toda la lluvia de la época húmeda.

Palabras claves: Caracterización de precipitaciones, periodos secos, periodos húmedos

\section{Introducción}

La ciudad de Quevedo al igual que el resto de la provincia de Los Ríos presenta anormal distribución temporal de las precipitaciones, el motivo de esta situación es que anualmente hay épocas con excesivas lluvias que provocan inundaciones, ocasionando pérdidas en el sector agrícola y temporadas en las que la ausencia de precipitaciones se considera sequía (Caicedo Camposano, Balmaseda Espinosa, \& Proaño Saraguro, 2015).

La producción agrícola del área de influencia de la Estación Meteorológica Pichilingue (EMP), se realiza a mayor escala en la estación seca, por ello es importante conocer el régimen de las precipitaciones de la zona, ya que éste suele ser un factor limitante en los rendimientos de las cosechas.

Estudios sobre caracterización de precipitaciones fueron realizados por (Prohaska, 1952; Canziani, Forte Lay, \& Troha, 1992; Ravelo, Sanz Ramos, \& Douriet Cárdenas, 2014) quienes caracterizaron los regímenes 
estacionales de precipitación en algunas localidades argentinas y de Sudamérica. Por otro lado (Naranjo, 2012), tuvo como prioridad la estimación de periodos secos y periodos húmedos en la cuenca de El Tajo en España.

Las precipitaciones en la zona de Quevedo se presentan únicamente en un determinado momento del año. El régimen de precipitaciones en algunas ciudades de la costa ecuatoriana como Manta, La Libertad, Guayaquil y Puerto Bolívar, se caracteriza por precipitaciones que van de diciembre a mayo; de esos meses los mayores volúmenes de precipitación los aportan febrero y marzo, luego de junio a noviembre las precipitaciones descienden por debajo del promedio mensual y en ciertos meses los valores se acercan a cero (Hernández \& Zambrano, 2007); (Gálvez \& Regalado, 2009).

Para la caracterización de precipitaciones es necesario identificar los periodos secos, para esto, en el ámbito de la hidrología se han generado índices de sequías, los cuales permiten puntualizar y comparar la influencia de estas, en cuencas hidrográficas, las mismas que tienen como consecuencias impactos económicos, sociales y medioambientales (Valiente, 2001).

Es imprescindible estudiar el régimen de precipitaciones, atender sus características y tendencias, y con los resultados que se obtengan, posteriormente se pueden aplicar estrategias para aprovechar eficientemente el agua (Sacchi, Dalla Marta, Costanzo, \& Coronel, 2002), sobre todo en la producción agrícola, lográndose con esto ajustar los calendarios de cultivo para obtener prácticas que vuelvan eficiente la agricultura de secano (Meseth \& Yu, 2014), práctica tradicional por falta de infraestructura de riego en la producción de maíz y frejol en la localidad de estudio.

El objetivo de este trabajo fue conocer el régimen de precipitaciones de la ciudad de Quevedo para contribuir al mejoramiento de los calendarios de cultivos de ciclo corto, puesto que, esta información es comúnmente requerida por agricultores de la zona y profesionales agrícolas, que laboran como asesores técnicos.

\section{Materiales y métodos}

La EMP está ubicada en el km 5 vía al Empalme en los predios de la Estación Experimental Tropical Pichilingue (EETP) del Instituto Nacional Autónomo de Investigaciones Agropecuarias (INIAP), en la ciudad de Quevedo, provincia de Los Ríos, la georeferenciación establecida por el Instituto Geográfico Militar Ecuatoriano son las coordenadas geográficas $01^{\circ}$ $06^{\prime} 00^{\prime \prime}$ de Latitud Sur y $79^{\circ} 27^{\prime} 42^{\prime \prime}$ de Longitud Oeste, altitud de 120 msnm. Esta estación se encuentra en funcionamiento y forma parte de la red 
de estaciones meteorológicas del Instituto Nacional de Meteorología e Hidrología (INAMHI).

Para la determinación de periodos secos y periodos húmedos, se empleó un método estadístico conformado a través de series de tiempo, las cuales pueden ser anuales o periódicas, estas últimas pueden ser desde estacionales hasta horarias (Naranjo, 2012).

Los registros que se emplearon para este trabajo fueron tratados como series univariadas por lo cual se calculó un conjunto de estadísticos tales como media, desviación estándar, coeficiente de variación, varianza, coeficiente de asimetría y coeficiente de curtosis. Para cada periodo seco y húmedo se estableció su duración, los periodos en que se presentaron, la periodicidad, el déficit o el exceso según sea el caso y la intensidad.

Los datos utilizados fueron de la precipitación acumulada anual (Tabla 1) provenientes de los registros del pluviómetro; esta información reposa en los archivos del INAMHI.

Tabla 1 Precipitaciones históricas mensuales

\begin{tabular}{|c|c|c|c|c|c|c|c|c|c|c|c|c|c|}
\hline $\begin{array}{l}\text { Meses } \\
\text { Años }\end{array}$ & Ene. & Feb. & Mar. & Abr. & May. & Jun. & Jul. & Ago. & Sep. & Oct. & Nov. & Dic. & Anual $\left(\mathrm{h}_{\mathrm{p}}\right)$ \\
\hline 1981 & 488,1 & 284,7 & 139,6 & 223,1 & 46,1 & 1,2 & 1,0 & 2,3 & 11,2 & 130,6 & 408,6 & 891,3 & 2627,8 \\
\hline 1983 & 119,4 & 552,1 & 512,3 & 284,6 & 7,6 & 67,0 & 2,4 & 4,9 & 13,1 & 5,3 & 19,0 & 199,6 & 1787,3 \\
\hline 1984 & 270,6 & 257,6 & 293,2 & 115,7 & 47,7 & 13,2 & 1,2 & 4,6 & 39,0 & 1,6 & 13,0 & 276,4 & 1333,8 \\
\hline 1985 & 625,2 & 370,6 & 199,7 & 393,6 & 40,4 & 0,7 & 1,7 & 3,5 & 5,6 & 69,0 & 9,1 & 108,9 & 1828,0 \\
\hline 1986 & 505,1 & 380,6 & 563,9 & 581,5 & 262,9 & 0,1 & 2,0 & 55,5 & 12,1 & 10,2 & 31,9 & 92,0 & 2497,8 \\
\hline 1988 & 459,4 & 487,7 & 471,9 & 483,8 & 146,7 & 15,6 & 7,8 & 0,7 & 1,6 & 30,5 & 13,9 & 114,6 & 2234,2 \\
\hline 1989 & 184,0 & 558,9 & 226,0 & 218,1 & 42,0 & 25,5 & 7,9 & 0,0 & 0,8 & 19,3 & 9,6 & 109,7 & 1401,8 \\
\hline 1990 & 292,6 & 653,2 & 352,9 & 250,1 & 46,6 & 8,1 & 1,1 & 0,3 & 0,8 & 6,4 & 28,3 & 214,6 & 1855,0 \\
\hline 1991 & 543,0 & 443,4 & 688,2 & 831,1 & 363,9 & 92,1 & 21,0 & 0,9 & 7,3 & 4,4 & 3,9 & 86,8 & 3086,0 \\
\hline 1992 & 427,0 & 656,4 & 459,4 & 521,2 & 80,8 & 36,0 & 1,4 & 5,3 & 6,8 & 26,6 & 0,8 & 176,2 & 2397,9 \\
\hline 1993 & 441,2 & 414,9 & 376,5 & 298,9 & 76,3 & 13,0 & 0,2 & 0,1 & 2,7 & 73,5 & 8,4 & 201,9 & 1907,6 \\
\hline 1994 & 439,8 & 266,6 & 192,6 & 475,4 & 66,4 & 15,1 & 19,2 & 9,0 & 2,1 & 10,6 & 31,7 & 58,9 & 1587,4 \\
\hline 1999 & 239,9 & 281,2 & 473,7 & 430,2 & 153,1 & 23,4 & 0,3 & 2,5 & 7,7 & 7,1 & 9,1 & 58,3 & 1686,5 \\
\hline 2000 & 618,1 & 288,2 & 192,9 & 507,1 & 188,5 & 1,5 & 2,5 & 0,0 & 1,1 & 0,3 & 11,8 & 56,1 & 1868,1 \\
\hline 2001 & 145,3 & 540,1 & 691,3 & 627,3 & 190,0 & 12,9 & 2,8 & 0,6 & 1,9 & 18,9 & 72,6 & 222,5 & 2526,2 \\
\hline 2002 & 446,6 & 386,7 & 269,1 & 344,4 & 170,4 & 23,6 & 34,8 & 8,8 & 1,1 & 73,1 & 53,3 & 187,0 & 1998,9 \\
\hline 2003 & 236,5 & 204,2 & 365,9 & 284,1 & 240,2 & 12,4 & 5,1 & 2,0 & 18,1 & 13,8 & 2,1 & 27,9 & 1412,3 \\
\hline 2004 & 227,0 & 245,7 & 192,5 & 462,0 & 6,2 & 0,9 & 3,7 & 0,5 & 2,0 & 1,3 & 3,4 & 108,0 & 1253,2 \\
\hline 2005 & 263,5 & 629,5 & 485,0 & 144,7 & 32,8 & 17,1 & 5,9 & 9,2 & 11,2 & 4,0 & 28,4 & 49,1 & 1680,4 \\
\hline 2006 & 242,9 & 272,9 & 371,2 & 407,6 & 116,8 & 29,7 & 19,8 & 0,7 & 0,5 & 1,4 & 21,7 & 55,7 & 1540,9 \\
\hline 2007 & 535,3 & 490,2 & 592,7 & 320,5 & 141,7 & 8,8 & 7,8 & 67,2 & 10,8 & 13,6 & 9,9 & 31,1 & 2229,6 \\
\hline 2008 & 301,5 & 288,4 & 381,1 & 201,0 & 140,0 & 13,5 & 1,4 & 0,6 & 0,3 & 6,0 & 0,1 & 65,0 & 1398,9 \\
\hline 2009 & 389,0 & 835,3 & 489,1 & 694,4 & 231,9 & 18,7 & 31,8 & 1,5 & 13,8 & 1,1 & 24,8 & 300,7 & 3032,1 \\
\hline 2010 & 369,6 & 490,5 & 144,1 & 725,7 & 9,9 & 48,4 & 47,3 & 1,1 & 4,2 & 4,2 & 4,8 & 150,6 & 2000,4 \\
\hline
\end{tabular}




\begin{tabular}{cccccccccccccc} 
Varianza & 36807,8 & 23788,5 & 49442,9 & 33352,7 & 31638,9 & 10086,6 & 11246,1 & 742,6 & 4677,7 & 1740,3 & 38475,9 & 29768,4 & 909569,4 \\
C. variación & 0,5 & 0,3 & 0,5 & 0,5 & 1,0 & 2,1 & 2,8 & 2,3 & 2,8 & 1,6 & 3,0 & 1,2 & 0,4 \\
C. Asimetría & 1,2 & 0,4 & 0,7 & 0,6 & 1,5 & 3,5 & 4,0 & 2,9 & 4,7 & 3,0 & 5,0 & 3,1 & 1,8 \\
C. Curtosis & 2,0 & $-0,3$ & 0,6 & $-0,1$ & 2,1 & 12,8 & 16,8 & 7,8 & 23,5 & 10,0 & 26,4 & 10,8 & 2,9 \\
\hline
\end{tabular}

\section{Resultados y Discusión}

A partir de los promedios para cada mes del año obtenidos en la Tabla 1, se establece la caracterización de las precipitaciones en el año como se muestra en la Figura 1, allí se observan con claridad los meses que conforman la estación húmeda o época lluviosa, coincidiendo este resultado con el comportamiento de las lluvias estudiadas por (Hernández \& Zambrano, 2007); (Gálvez \& Regalado, 2009).

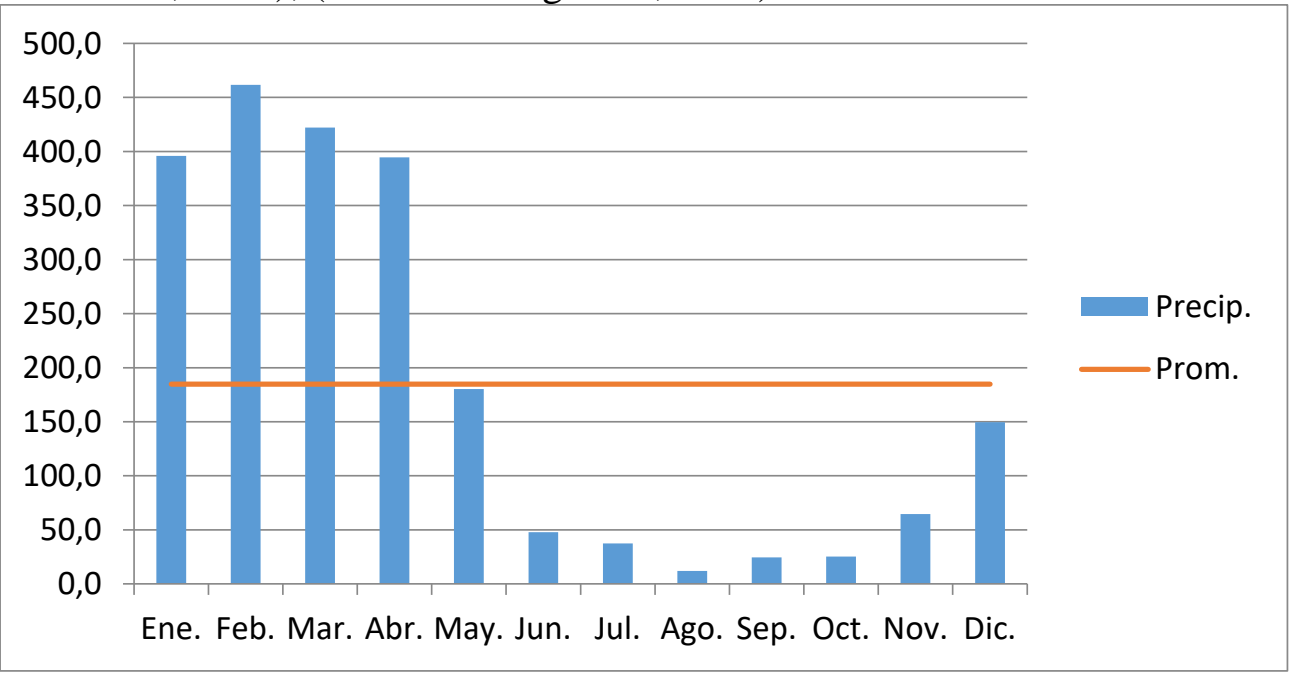

Figura 1. Histograma de las precipitaciones de enero a diciembre en la EMP Fuente: Elaboración propia

En términos estadísticos, la EPM cuenta con una longitud bastante amplia, lo cual garantiza el certero reconocimiento de la ocurrencia de periodos secos y periodos húmedos.

Con los valores de la precipitación anual acumulada durante 35 años de registro se construye la Figura 2, donde en función del trazado del promedio, se muestran los años con déficits y excedentes de precipitaciones. Consiguientemente la Tabla 2 contiene la serie de diferencias $D_{p},(\mathrm{~mm})$, considerando como nivel de truncamiento la lluvia promedio anual $\mathrm{X}_{0}=$ $2216,3 \mathrm{~mm}$. Esto se obtuvo restando a cada registro anual el valor promedio de los acumulados anuales. Los resultados negativos corresponden a años secos, mientras que los positivos son años húmedos. 


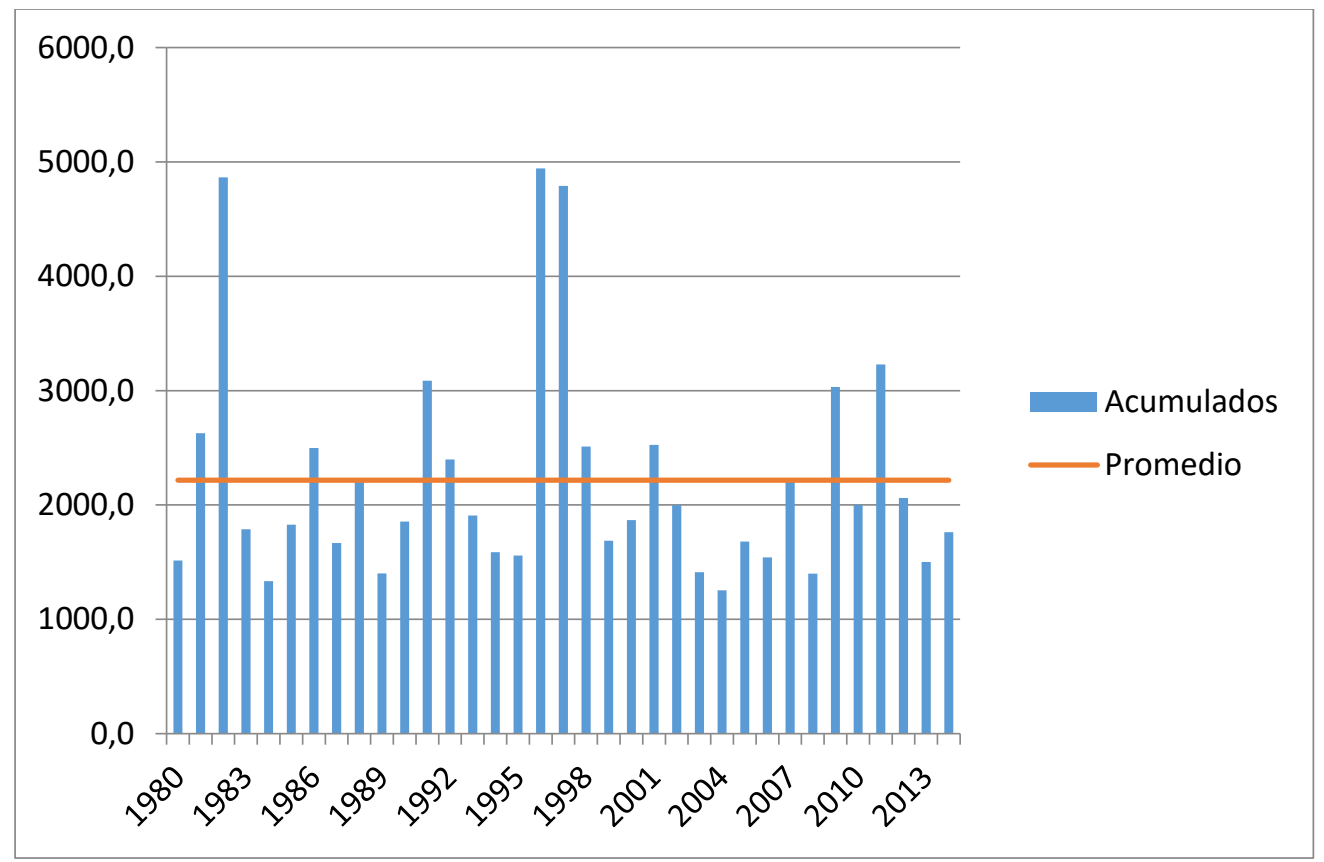

Figura 2. Histograma de precipitaciones anuales

Fuente: Elaboración propia

Tabla 2 Serie de diferencias $D_{p}$ de la EMP

\begin{tabular}{cccc}
\hline Año & $D_{p}$ & Año & $D_{p}$ \\
\hline 1980 & $-702,1$ & 1998 & 293,7 \\
1981 & 411,5 & 1999 & $-529,8$ \\
1982 & 2647,9 & 2000 & $-348,2$ \\
1983 & $-429,0$ & 2001 & 309,9 \\
1984 & $-882,5$ & 2002 & $-217,4$ \\
1985 & $-388,3$ & 2003 & $-804,0$ \\
1986 & 281,5 & 2004 & $-963,1$ \\
1987 & $-549,0$ & 2005 & $-535,9$ \\
1988 & 17,9 & 2006 & $-675,4$ \\
1989 & $-814,5$ & 2007 & 13,3 \\
1990 & $-361,3$ & 2008 & $-817,4$ \\
1991 & 869,7 & 2009 & 815,8 \\
1992 & 181,6 & 2010 & $-215,9$ \\
1993 & $-308,7$ & 2011 & 1013,0 \\
1994 & $-628,9$ & 2012 & $-155,3$ \\
1995 & $-659,0$ & 2013 & $-715,2$ \\
1996 & 2726,2 & 2014 & $-454,4$ \\
1997 & 2574,6 & & \\
\hline
\end{tabular}


Tabla 3 Características de periodos secos identificados en la EMP

\begin{tabular}{|c|c|c|c|c|c|}
\hline $\begin{array}{c}\text { Periodo } \\
\text { Seco }\end{array}$ & $\begin{array}{c}\text { Duración } \\
\text { (años) }\end{array}$ & Periodo & $\begin{array}{c}\text { Periodicidad } \\
\text { (años) }\end{array}$ & $\begin{array}{c}\text { Déficit } \\
(\mathrm{mm})\end{array}$ & $\begin{array}{c}\text { Intensidad } \\
(\mathrm{mm} / \mathrm{año})\end{array}$ \\
\hline 1 & 1 & $1980-1980$ & & 702,1 & 702,1 \\
\hline 2 & 3 & $1983-1985$ & 3 & 1699,9 & 566,6 \\
\hline 3 & 1 & $1987-1987$ & 4 & 549,0 & 549,0 \\
\hline 4 & 2 & $1989-1990$ & 2 & 1175,9 & 587,9 \\
\hline 5 & 3 & $1993-1995$ & 4 & 1596,7 & 532,2 \\
\hline 6 & 2 & $1999-2000$ & 6 & 878,1 & 439,0 \\
\hline 7 & 5 & $2002-2006$ & 3 & 3731,9 & 746,4 \\
\hline 8 & 1 & $2008-2008$ & 6 & 817,4 & 817,4 \\
\hline 9 & 1 & $2010-2010$ & 2 & 215,9 & 215,9 \\
\hline 10 & 3 & $2012-2014$ & 2 & 1325,0 & 441,7 \\
\hline Media & 2,1 & & 3,8 & 1263,0 & 573,0 \\
\hline Des. Est. & 1,2 & & 1,5 & 931,5 & 164,3 \\
\hline Coe. Var. & 0,6 & & 0,4 & 0,7 & 0,3 \\
\hline
\end{tabular}

Tabla 4 Características de periodos húmedos identificados en la EMP

\begin{tabular}{|c|c|c|c|c|c|}
\hline $\begin{array}{c}\text { Periodo } \\
\text { Húmedo }\end{array}$ & $\begin{array}{c}\text { Duración } \\
\text { (años) }\end{array}$ & Periodo & $\begin{array}{c}\text { Periodicidad } \\
\text { (años })\end{array}$ & $\begin{array}{c}\text { Excedente } \\
(\mathrm{mm})\end{array}$ & $\begin{array}{c}\text { Intensidad } \\
(\mathrm{mm} / \text { año) }\end{array}$ \\
\hline 1 & 2 & $1981-1982$ & & 3059,3 & 1529,7 \\
\hline 2 & 1 & $1986-1986$ & 4 & 281,5 & 281,5 \\
\hline 3 & 1 & $1988-1988$ & 2 & 17,9 & 17,9 \\
\hline 4 & 2 & $1991-1992$ & 4 & 1051,2 & 525,6 \\
\hline 5 & 3 & $1996-1998$ & 6 & 5594,4 & 1864,8 \\
\hline 6 & 1 & $2001-2001$ & 3 & 309,9 & 309,9 \\
\hline 7 & 1 & $2007-2007$ & 6 & 13,3 & 13,3 \\
\hline 8 & 1 & $2009-2009$ & 2 & 815,8 & 815,8 \\
\hline 9 & 1 & $2011-2011$ & 2 & 1013,0 & 1013,0 \\
\hline Media & 1,4 & & 3,6 & 1350,7 & 707,9 \\
\hline Des. Est. & 0,7 & & 1,6 & 1738,8 & 619,2 \\
\hline Coe. Var. & 0,5 & & 0,4 & 1,3 & 0,9 \\
\hline
\end{tabular}

De los registros examinados fueron detectados 10 periodos secos y 9 periodos húmedos.

Un periodo seco se presenta con una periodicidad de 3,8 años y una duración esperada de 2,1 años; igualmente un periodo húmedo se presenta con periodicidad de 3,6 años y su duración es de 1,4 años, confirmándose lo expresado por Naranjo (2012) quien manifiesta que los mencionados periodos son cíclicos.

La probabilidad de que se ocurra un periodo seco con una duración de 1, 2, 3, 4 y 5 años es de 50\% (5/10), 40\%, 30\%, 20 y 10\%. 
El periodo seco máximo registrado ocurrió entre 2002 y 2006, con un valor acumulado de 3731,9 mm, una intensidad de $746,4 \mathrm{~mm} /$ año y una desviación estándar en relación a la lluvia promedio anual del 34 \%.

Con la intensidad del periodo seco máximo registrado se puede determinar que la lámina disponible media anual entre 2002 y 2006 fue equivalente a: $h_{p}=2216,3-746,4=1469,9 \mathrm{~mm}$.

La máxima intensidad registrada en el periodo seco se presentó entre 2008 con 817,4 mm/ año, con una lámina disponible media anual de $\mathrm{h}_{\mathrm{p}}=$ $2216,3-817,4=1398,9 \mathrm{~mm}$, y una desviación estándar respecto a la lluvia media anual del $37 \%$.

La media del déficit acumulado es de 1263,0 mm, para su duración media se tiene una intensidad $573,0 \mathrm{~mm} /$ año. La lámina disponible media anual es de $h_{p}=2216,3-1263,0=953,3 \mathrm{~mm}$, o sea $43 \%$ por debajo de la lluvia media anual.

Los resultados de la Tabla 3 muestran que un periodo húmedo de lluvia se presenta cada 3,6 años, con una duración media esperada de 1,4 años.

La probabilidad de que se presente un periodo húmedo con una duración de 1, 2 y 3 años es de 33\%, 22\% y 11\%.

El máximo periodo húmedo registrado ocurrió entre 1996 y 1998 con un valor acumulado de 5594,4 mm e intensidad de 1864,8 mm/ año.

Con la intensidad detectada se puede establecer que la lámina disponible media anual en el periodo húmedo ocurrido entre 1996 a 1998 es: $\mathrm{h}_{\mathrm{p}}=2216,3+11864,8=7459,2 \mathrm{~mm}$.

La máxima intensidad registrada ocurrió en el periodo 1996 a 1998) con 1864,8 mm/ año, y una lámina medio anual disponible de $\mathrm{h}_{\mathrm{p}}=2216,3+$ $1864,8=4081,1 \mathrm{~mm}$. El excedente acumulado promedio es de 1350,7 mm, para su duración promedio se detectó una intensidad 709,9 mm/ año. La lámina promedio disponible en el año es de $h_{p}=2216,3+707,9=2924,2$ $\mathrm{mm}$, o sea $32 \%$ por arriba de la precipitación promedio anual.

\section{Conclusion}

Los meses que definen la estación húmeda son diciembre, enero, febrero, marzo, abril y mayo, mientras que los meses de junio a noviembre vienen siendo los que conforman la estación seca.

La lámina disponible promedio de lluvia acumulada determinada para los periodos seco y húmedo tiene los valores siguientes: $h_{p}$ (déficit) $=953,3$ $\mathrm{mm}, \mathrm{h}_{\mathrm{p}}($ media $)=2216,3 \mathrm{~mm}, \mathrm{~h}_{\mathrm{p}}($ excedente $)=2924,2 \mathrm{~mm}$.

En función de la periodicidad con que se presentan los periodos seco y húmedo, se puede decir que estos muestran ciclicidad. El periodo seco más crítico tuvo una duración de 4 años y se presentó de 2002 a 2006 acumulando un déficit de $3731,9 \mathrm{~mm}$, que representa el $168 \%$ de la lluvia 
media anual, situación que enfatiza la gravedad de las sequias en la zona estudiada.

Los cultivos de maíz y frejol deberían sembrarse desde el mes de diciembre, de esa manera estas especies vegetales aprovecharán toda la precipitación de la estación húmeda.

\section{References:}

1. Caicedo Camposano, O., Balmaseda Espinosa, C., \& Proaño Saraguro, J. (2015). Programación del riego del banano (Musa paradisiaca) en finca San José 2, Los Ríos, Ecuador. Revista Ciencias Técnicas Agropecuarias, 24(2), 18-22.

2. Canziani, O., Forte Lay, J., \& Troha, A. (1992). Estacionalidad de las precipitaciones en el territorio continental argentino. Geoacta, 19, 2136.

3. Gálvez, H., \& Regalado, J. (2009). Comportamiento de las precipitaciones en la costa ecuatoriana en el 2008. Acta Oceanográfica del Pacífico, 15(1), 19-26.

4. Hernández, F., \& Zambrano, E. (2007). Inicio, duración y término de la estación lluviosa en cinco localidades de la costa ecuatoriana. Acta Oceanográfica del Pacífico, 14(1), 7-11.

5. Meseth, E., \& Yu, J. (2014). Mejora en los calendarios de cultivo para agricultura de secano en ceja de selva. Scientia Agropecuaria, 5(4), 187-197.

6. Naranjo, M. F. (2012). Estimación de períodos húmedos y secos en la Hidrología. Revista de Educación en Ciencias e Ingeniería (UAM-I), 85, 56-72.

7. Prohaska, F. (1952). Regímenes estacionales de precipitación de Sudamérica y mares vecinos (desde $15^{\circ} \mathrm{S}$ hasta Antártida). Meteoros, 2, 66-100.

8. Ravelo, A. C., Sanz Ramos, R., \& Douriet Cárdenas, J. C. (2014). Detección, evaluación y pronóstico de las sequías en la región del Organismo de Cuenca Pacífico Norte, México. Agriscientia, 31(1), 11-24.

9. Sacchi, O., Dalla Marta, N., Costanzo, M., \& Coronel, A. (2002). Caracterización de las precipitaciones en la localidad de Zavalla. Revista de Investigaciones de la Facultad de Ciencias Agrarias UNR, 2(2), 91-103.

10. Valiente, Ó. M. (2001). Sequía: definiciones, tipologías y métodos de cuantificación. Investigaciones Geográficas, (26), 59-80. 